\title{
Design and Fabrication of Temperature Sensor for Weather Monitoring System using Micro Electro Mechanical System Technology
}

\author{
VEERAMANI P., ${ }^{1 *}$ VIMALA JULIET A., ${ }^{1}$ SAM JEBAKUMAR J., ${ }^{1}$ and JAGADISH R., ${ }^{2}$ \\ 1Department of Electronics and Instrumentation Engineering, SRM Institute of Science and \\ Technology, Kattankulathur, Kanchipuram, India. \\ ${ }^{2}$ Research Institute, SRM Institute of Science and Technology, Kattankulathur, Kanchipuram, India. \\ ${ }^{*}$ Corresponding author E-mail: veerasrm.1993@gmail.com
}

http://dx.doi.org/10.13005/ojc/340537

(Received: March 19, 2018; Accepted: August 17, 2018)

\begin{abstract}
In this paper, Micro Electro Mechanical System (MEMS) based temperature sensor is designed and fabricated for weather monitoring system at troposphere level. In this design we have used meander shape, because it is easy to vary the length. We have optimized the length in this design. Due to certain advantages like low cost, easily available, high melting and boiling point, molybdenum material is used for fabrication of this design. The four meander type temperature sensors are designed with various dimensions of sizes in $6.7 \mathrm{~mm} \times 4 \mathrm{~mm}, 9.5 \mathrm{~mm} \times 4 \mathrm{~mm}, 5.2 \mathrm{~mm} \times 4 \mathrm{~mm}, 6.5 \mathrm{~mm} \times 4 \mathrm{~mm}$. The Temperature Coefficient of Resistance (TCR) values for four various sensors mentioned above are $3.4 \times 10^{-4} \mathrm{C}^{-1}, 3.7 \times 10^{-4} \mathrm{C}^{-1}, 7.0 \times 10^{-4} \mathrm{C}^{-1}, 7.5 \times 10^{-4} \mathrm{C}^{-1}$. For radiosonde applications the sensor must have high sensitivity, high degree of accuracy, good linearity and with better TCR values. The experimental results are better for dimension $6.7 \mathrm{~mm} \times 4 \mathrm{~mm}$ for all characteristics mentioned above. The practical results are compared with the theoretical values.
\end{abstract}

Keywords: Temperature sensor, Micro Electro Mechanical System, Radiosonde.

\section{INTRODUCTION}

Micro Electro Mechanical Systems (MEMS) is a technology to develop micro devices and micro systems. MEMS are used for miniaturization of sensors. MEMS components are generally in size from micrometers to millimeters. The reliability of these sensors have been increased considerably by integrating them with IC technology ${ }^{1,2}$. MicroElectro Mechanical System fabrication technology is employed to reduce size of the sensor, cost of electronic devices in various applications, it is the important thing that the electronic device must be stable, portable, and flexible. To design a temperature sensor for weather monitoring in radiosonde application using MEMS technology. The temperature sensor was designed to measure the troposphere level. Nowadays the sensors used in radiosonde application are big in size. The temperature sensor is designed to reduce the size 
and cost of the sensor. In order to sense these conditions, an appropriate sensing method has to been utilized in order to achieve accurate outputs in a study manner.

Basically sensors can be classified into two types. One is active sensor and another one is passive sensor. Active sensor is self generating. Passive sensor is modulating. The Active sensor converts input energy into output signal and it does not require any external supply. The Passive sensor requires additional power source to converts input energy into an output signal. The function of a temperature sensor can be classified into three different modes. They are physical, mechanical and optical. However, our target is to obtain electrical outputs. The electrical sensor is used for measuring the temperature. One of the simplest and easiest electrical technologies for measuring temperature is by using resistive measurement. Resistance Temperature Detector (RTD) and thermistor is the temperature sensor which changes electrical resistance with temperature. In this method works on the fact that resistivity of the material is temperature dependent. The temperature of the RTD ranges from $-200^{\circ} \mathrm{C}$ to $600^{\circ} \mathrm{C}$ with an excellent accuracy over a wide temperature range $\mathrm{e}^{3,4}$.

\section{Methodology \\ Theory of temperature sensor}

The resistance of the metal is described as,

$R=\rho L / A$

If temperature of the RTD varies linearly, then the relationship between the measured resistance and the temperature chang $\mathrm{e}^{5,6,7} \mathrm{can}$ be expressed as

$$
R_{t}=R_{0}\left[1+\alpha\left(t-t_{0}\right)\right]
$$

Where,

$R_{t}$ is the resistance at $t^{\circ} \mathrm{C}$

$\mathrm{R}_{0}$ is the resistance at $0^{\circ} \mathrm{C}$

$\alpha$ is the temperature coefficient of resistance $t$ is the temperature to be measured

$\mathrm{t}_{0}$ is the initial temperature

\section{Design of micro temperature sensor}
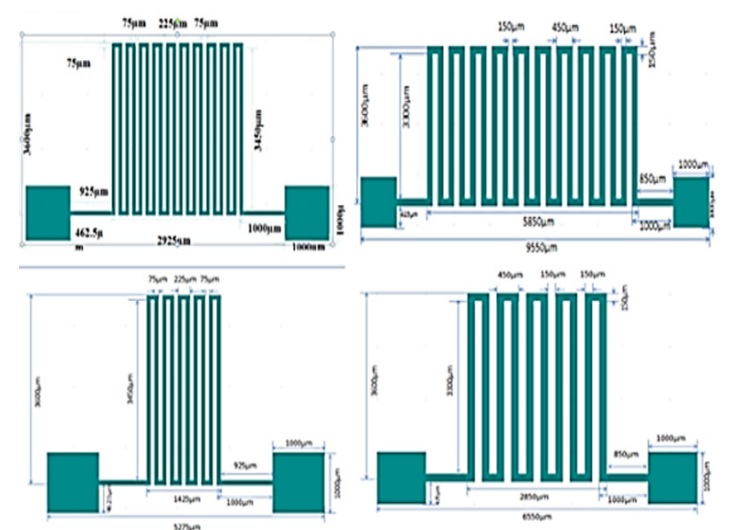

Fig. 1. Design of Temperature Sensors

The temperature sensor has been analyzed with different dimensions. When its length increases the resistance value will also increase proportionally. The important thing to be considered is Temperature Coefficient of Resistance (TCR). Where TCR is defined as the resistance change factor per degree Celsius of temperature change. By changing the temperature the resistance value is recorded. The TCR value was calculated based on these recorded values.

\section{Design of temperature sensors using comsol}

A Multiphysics numerical stimulator COMSOL was used to design and stimulate the sensor design. In this section the design aspects is discussed. Joule heating module is selected as the physics for the model. Fixed current and temperature are assumed at the end of the heater. The current supplied is in few hundred microns (0.07A). The electrical current converted into heat flows through resistor 8,9 . The work presents the corresponding electrical potential of four temperature sensors.
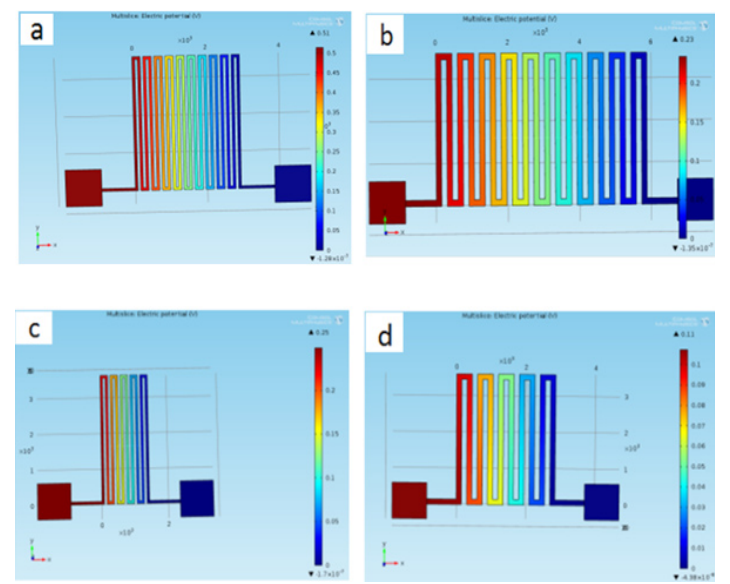

Fig. 2. Electric Potential over sensor

(a) $(6.7 \mathrm{~mm} \times 4 \mathrm{~mm})$, (b) $(9.5 \mathrm{~mm} \times 4 \mathrm{~mm})$, (c) $(5.2 \mathrm{~mm} \times 4 \mathrm{~mm})$, (d) $(6.5 \mathrm{~mm} \times 4 \mathrm{~mm})$ 
Figure 2 shows how the electrical potential is distributed for four different dimensions. Fig. 3 shows dimensions of sensor. Whenever the length increases the resistance value also increases. Fig. 4 shows relationship between the resistance and input temperature of molybdenum based temperature sensor and observed resistance of the sensor increase linearly with increase in input temperature for the specified ranges.
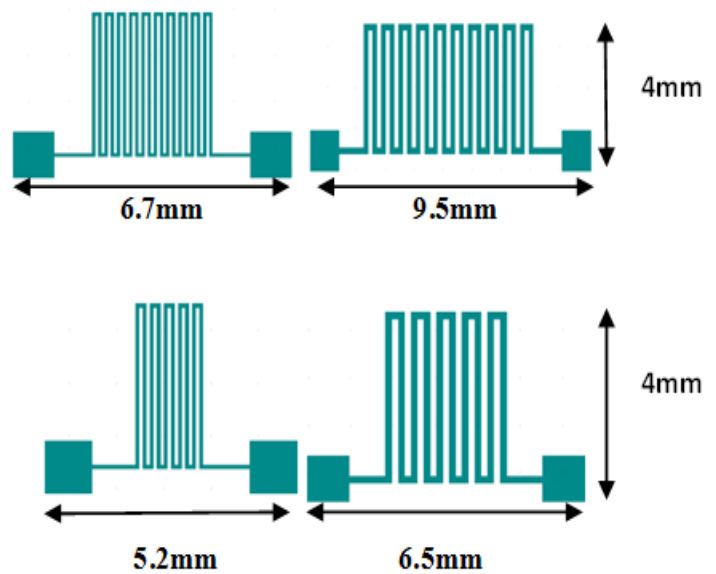

Fig. 3. Dimension of Temperature Sensor

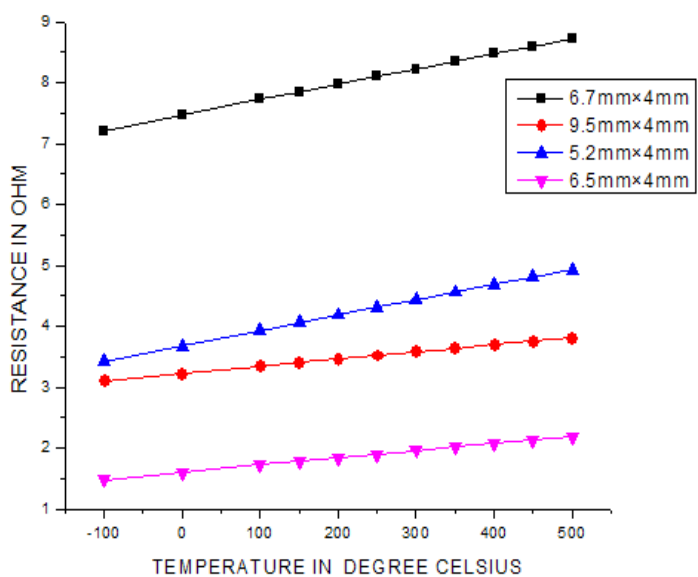

Fig. 4. Temperature vs. Resistance

The temperature sensor is of size in $6.7 \mathrm{~mm} \times 4 \mathrm{~mm}$ which linearly increases in resistance value (7.208 to $8.731 \Omega$ ) according to the input temperature $\left(-100\right.$ to $\left.500^{\circ} \mathrm{C}\right)$. It works on the principle of resistance temperature detector. The temperature coefficient of resistance is $3.4 \times 10^{-4} \mathrm{C}^{-1}$. The sensor is of size $9.5 \mathrm{~mm} \times 4 \mathrm{~mm}$ which linearly increases in resistance (3.104 to $3.813 \Omega$ ) according to the input temperature $\left(-100\right.$ to $\left.500^{\circ} \mathrm{C}\right)$. The temperature coefficient of resistance is $3.7 \times 10^{-4} \mathrm{C}^{-1}$. The resistance of the sensor is directly proportional to the length of the sensing material molybdenum. The sensor is of size $5.2 \mathrm{~mm} \times 4 \mathrm{~mm}$ which linearly increases in resistance value(3.416 to $4.929 \Omega$ ) according to the input temperature $\left(-100\right.$ to $\left.500^{\circ} \mathrm{C}\right)$. The temperature coefficient of resistance is $7.0 \times 10^{-4} \mathrm{C}^{-1}$. The sensing material consists of size of $75 \mu \mathrm{m}$. The temperature sensor has $6.5 \mathrm{~mm} \times 4 \mathrm{~mm}$ which linearly increases in resistance (1.482 to $2.191 \Omega$ ) according to the input temperature $\left(-60\right.$ to $\left.60^{\circ} \mathrm{C}\right)$. The temperature coefficient of resistance is $7.5 \times 10^{-4} \mathrm{C}^{-1}$.

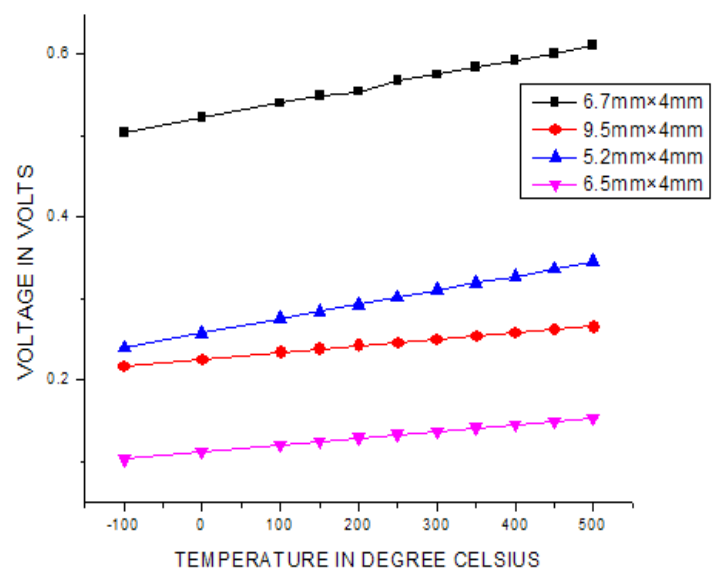

Fig. 5. Temperature vs. Voltage

In Figure 5, the temperature sensor consists is of size $6.7 \mathrm{~mm} \times 4 \mathrm{~mm}$ which linearly increases in voltage value ( 0.504 to $0.611 \mathrm{~V}$ ) according to the input temperature $\left(-100\right.$ to $\left.500^{\circ} \mathrm{C}\right)$. When passing a small amount of current 0.07 A to RTD generates a voltage across the RTD. By measuring the voltage the resistance and temperature value is determined.

The sensor has $9.5 \mathrm{~mm} \times 4 \mathrm{~mm}$ which is linearly increases voltage value $(0.217$ to 0.266 $\mathrm{V})$ according to the input temperature (-100 to $500^{\circ} \mathrm{C}$ ). It is gradually increases according to the input temperature. The sensor consists of size in $5.2 \mathrm{~mm} \times 4 \mathrm{~mm}$ which linearly increases in voltage value ( 0.239 to $0.345 \mathrm{~V}$ ) according to the input temperature $\left(-100\right.$ to $\left.500^{\circ} \mathrm{C}\right)$. It gradually increases according to the input temperature. The sensor has $6.5 \mathrm{~mm} \times 4 \mathrm{~mm}$ which is linearly increases in voltage value $(0.103$ to $0.153 \mathrm{~V}$ ) according to the input temperature $\left(-100\right.$ to $\left.500^{\circ} \mathrm{C}\right)$.

\section{Theoretical calculation}

According to Callender-Van Dusen equation, the RTDs are used to measure temperature by correlating the resistance. This correlation between 
the resistance and temperature of RTD is described by a Callender-Van Dusen equation.

The Callender-Van Dusen is an equation to determine resistance and temperature relationship at both positive and negative temperatures.

At positive temperature

$\mathrm{R}_{\mathrm{t}}=\mathrm{R}_{0}\left[1+\mathrm{At}+\mathrm{Bt}^{2}\right]$

At Negative temperature

$R_{t}=R_{0}\left[1+A t+B t^{2}+C(t-100)^{3}\right]$

Where A, B, C are Callender Van Dusen constants. The alpha, beta and delta are constants to determine the constant values of $\mathrm{A}, \mathrm{B}$ and $\mathrm{C}$. Equation (4) is typically used for temperature below $0^{\circ} \mathrm{C}$. Where equation (3) is used for temperature above $0^{\circ} \mathrm{C}$. When below $0^{\circ} \mathrm{C}$ is used in equation (3) the error is calculated resistance value. So we can use equation (4) for below $0^{\circ} \mathrm{C}^{10-12}$.

According to the calculation of each temperature sensor the resistance value is increased linearly with input temperature and the sensor $6.7 \mathrm{~mm} \times 4 \mathrm{~mm}$ has high sensitivity. The resistance and voltage value is calculated according to the temperature change.

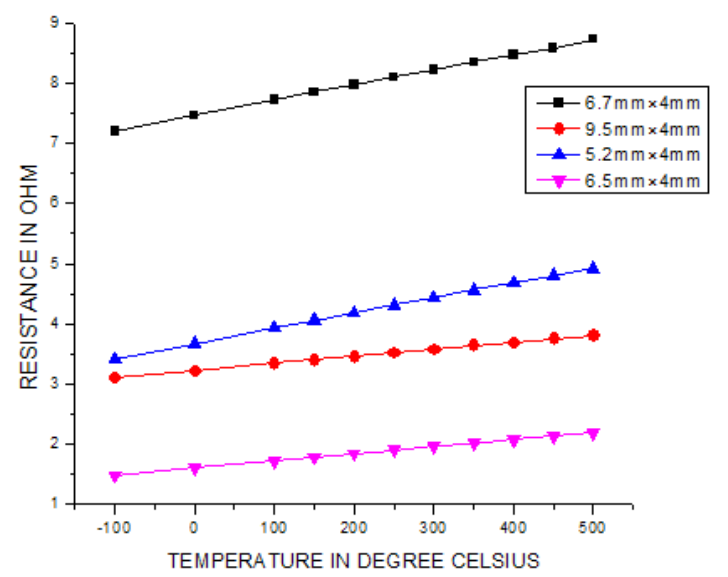

Fig. 6. Temperature vs. Resistance

According to Callender Van Dusen equation, the positive and negative temperature has different formula to calculate the resistance value. In Fig. 6 , the sensor $6.7 \mathrm{~mm} \times 4 \mathrm{~mm}$ shows that resistance $(7.20$ to $8.73 \Omega$ ) is increased linearly according to the input temperature $\left(-100\right.$ to $\left.500^{\circ} \mathrm{C}\right)$. The size of the sensor is $9.5 \mathrm{~mm} \times 4 \mathrm{~mm}$ which linearly increases in resistance (3.10 to $3.81 \Omega$ ) according to the temperature (-100 to $500^{\circ} \mathrm{C}$ ). When compared to $6.7 \mathrm{~mm} \times 4 \mathrm{~mm}$ it has lower resistance value. The $5.2 \mathrm{~mm} \times 4 \mathrm{~mm}$ sensor which shows linearly increases resistance (3.41 to $4.92 \Omega$ ) according to the input temperature ( -100 to $\left.500^{\circ} \mathrm{C}\right)$. The resistance is linearly increases $(1.482$ to $2.19 \Omega$ ) according to the input temperature $(-60$ to $60^{\circ} \mathrm{C}$ ) on sensor $6.5 \mathrm{~mm} \times 4 \mathrm{~mm}$. The TCR value is $7.0 \times 10^{-4} \mathrm{C}^{-1}$. So the resistance change is good according to degree temperature changes and sensitivity is good in $6.7 \mathrm{~mm} \times 4 \mathrm{~mm}$.

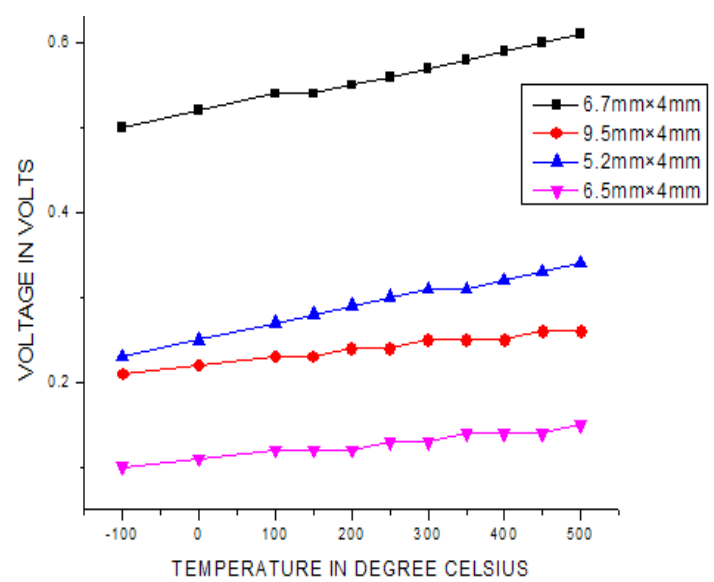

Fig. 7. Temperature vs. Resistance

In Fig. 7, the temperature sensor consists of size $6.7 \mathrm{~mm} \times 4 \mathrm{~mm}$ which linearly increases voltage value $(0.50$ to $0.61 \mathrm{~V})$ according to the input temperature. The sensor consists of size $9.5 \mathrm{~mm} \times 4 \mathrm{~mm}$ which linearly increases voltage value $(0.217$ to $0.266 \mathrm{~V})$ according to the input temperature. The sensor consists of size in $5.2 \mathrm{~mm} \times 4 \mathrm{~mm}$ which linearly increases voltage value $(0.23$ to $0.34 \mathrm{~V})$ according to the input temperature. The sensor which as $6.5 \mathrm{~mm} \times 4 \mathrm{~mm}$ which linearly increases voltage ( 0.10 to $0.15 \mathrm{~V}$ ) according to the input temperature. The small amount of current through a sensor generates a voltage across the sensor.

\section{EXPERIMENTAL}

\section{Fabrication of mask}

The laser writer Heidelberg MPG 501 was used for photo mask fabrication with commercially available chrome photo mask blank. A $390 \mathrm{~nm}$ laser was used with the writing speed of $50 \mathrm{~mm}^{2} /$ minute. The photomask layout for the micro fabrication of temperature sensor was designed using CleWin software. The fabricated photomask is shown in Fig. 9 with four different sensing arm length variations. 
Choice of Substrate and Photoresist materials

Silicon, being a good structural material with ideal semiconducting properties and mechanical stability was chosen as the substrate. Silicon wafers are annealed at $1000^{\circ} \mathrm{C}$ for $30 \mathrm{~min}$. in oxygen atmosphere to form an oxide layer on the surface.

ARP-3250, a positive photoresist (ALLRESIST, GmbH, Germany) was chosen for the preparation of resist template. ARP-3250 shows better integrity and resolution of the shapes for micro devices ${ }^{13}$. The photoresist was spin coated (SpinNXGP1) at $4000 \mathrm{rpm}$ for $30 \mathrm{sec}$. to grow $\sim 8 \mu \mathrm{m}$ thick resist. The evaporation of excess solvent was done by prebaking the resist coated substrates at $90^{\circ} \mathrm{C}$ for 8 minutes.

\section{Fabrication of resist template}

The desired patterns in the photomask were transferred to the resist by utilizing ultraviolet radiation. The photo mask was placed intact with resist coated silicon substrates. The UV exposure was done using OAI, USA flood exposure tool, model 0 . The UV was exposed with the controlled power of $10 \mathrm{~mW} / \mathrm{cm}^{2}$ for a time period of $25 \mathrm{~s}$. On completion of the UV exposure, the photo resist was developed using developer solution. The solution was the mixture of AR300-26:DI water in the ratio of 1:5. The development was carried out for a maximum duration of $200 \mathrm{~s}$. The desired sensor design thus transferred to the photoresist acts as template (shown in Fig. 11) for subsequent processes ${ }^{14}$.

\section{Deposition of molybdenum}

The molybdenum was deposited using DC sputtering process. The base pressure of chamber was $5 \times 10^{-5}$ mbar. Under Argon gas supply, the molybdenum was coated for $3 \mathrm{~min}$. with the working pressure of $8 \times 10^{-2}$ mbar. The optical image of deposited molybdenum on the photoresist template is shown in Figure 12.

\section{Resist removal}

The deposition of molybdenum was followed by resist removal process. Resist remover AR300-76 was used to remove the sacrificial resist. Removal process was done for $3-5$ seconds in ultra-bath sonicator at near room temperature. After resist removal the substrate was cleaned using DI water for several times. The optical microscope images of the temperature sensor fabricated on the $\mathrm{SiO}_{2}$ substrate is shown in Fig. 13. Repeated washing with DI water was done to remove residual part of resist from the surface.

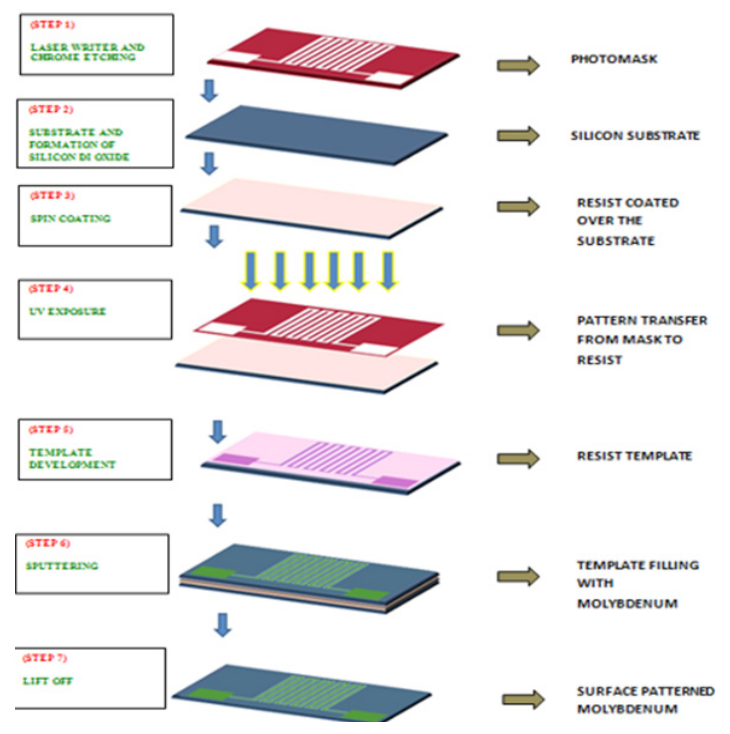

Fig. 8. Fabrication process of temperature sensor RESULTS AND DISCUSSION

The micro-temperature sensor (shown in Fig. 14) with sensing arm of $9.5 \mathrm{~mm} \times 4 \mathrm{~mm}$ along with contact pads was fabricated using microlithography technique.

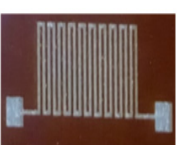

(a)

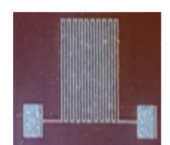

(b)

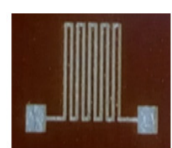

(c)

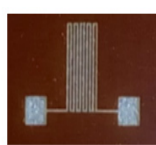

(d)
Fig. 9. Photomasks with variation in sensing arms (a) $9.5 \mathrm{~mm} \times 4 \mathrm{~mm}$ (b) $6.7 \mathrm{~mm} \times 4 \mathrm{~mm}$ (c) $6.5 \mathrm{~mm} \times 4 \mathrm{~mm}$ (d) $5.2 \mathrm{~mm} \times 4 \mathrm{~mm}$

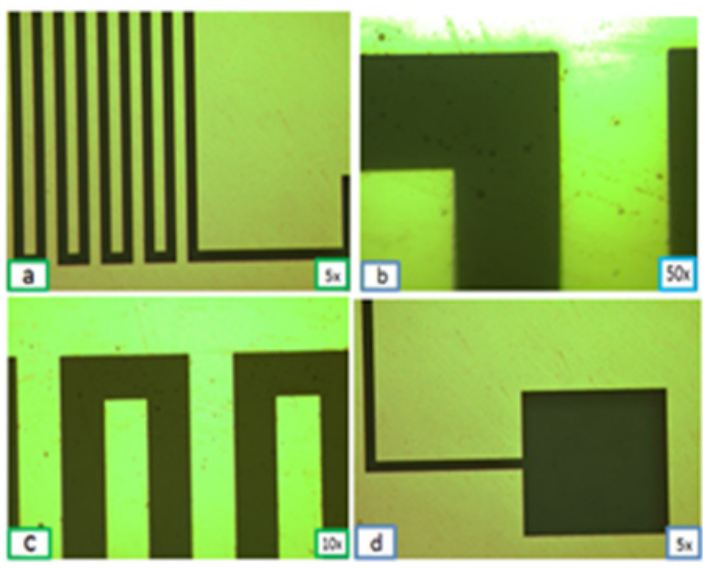

Fig. 10. Optical microscope images of $9.5 \mathrm{~mm} \times 4 \mathrm{~mm}$ photomask at different magnifications 


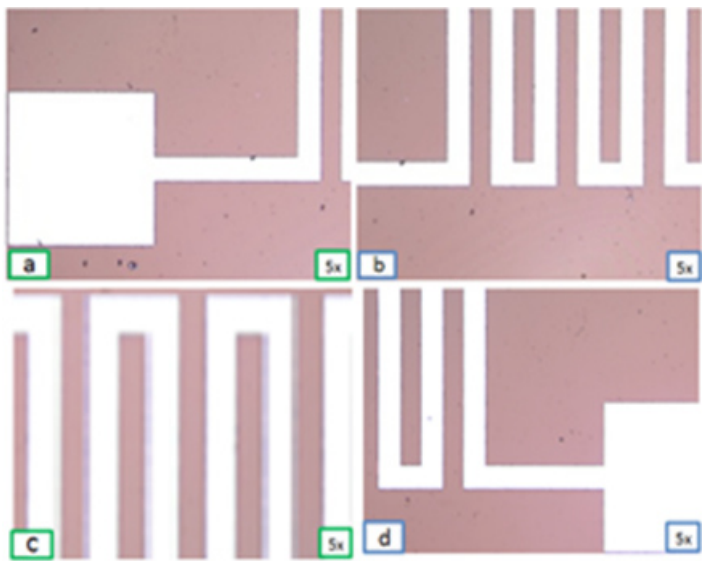

Fig. 11. Photoresist template of temperature sensor

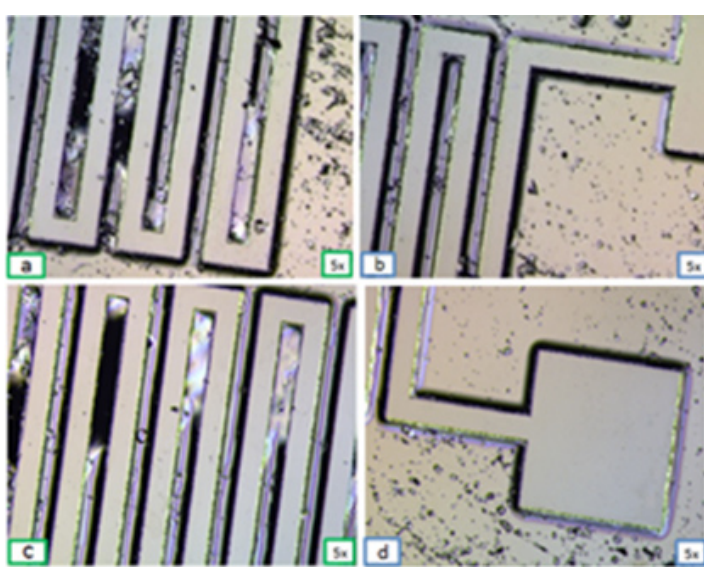

Fig. 12. Deposited molybdenum over the resist template

\section{CONCLUSION}

The novelty of this work is to reduce the size of the sensor in a radiosonde device by using MEMS based devices. The results obtained in comsol multiphysics software for the sensors $6.7 \mathrm{~mm} \times 4 \mathrm{~mm}$ and $5.2 \mathrm{~mm} \times 4 \mathrm{~mm}$ shows that the resistance changes linearly for a linear change in temperature and the TCR value are $3.4 \times 10^{-4} \mathrm{C}^{-1}$ and $7.0 \times 10^{-4} \mathrm{C}^{-1}$ respectively. The sensor $6.7 \mathrm{~mm} \times$ $4 \mathrm{~mm}$ has high resistance change according to the input temperature and sensitivity is also higher.

The molybdenum based temperature sensor was designed successfully using comsol multiphysics. The fabrication of $9.5 \mathrm{~mm} \times 4 \mathrm{~mm}$ sensor was done experimentally. A vacuum environment setup is made to measure the change in resistance of

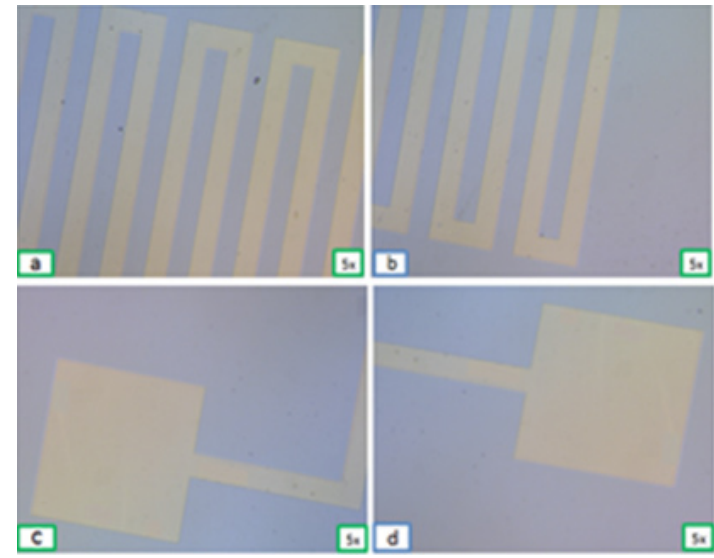

Fig. 13. Optical microscope images of the fabricated temperature sensor after complete removal of sacrificial resist

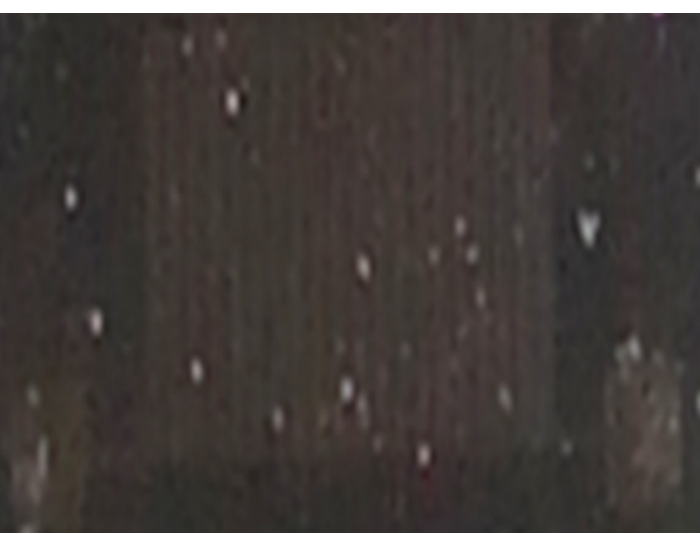

Fig. 14. Photograph of fabricated micro temperature sensor the temperature sensor as a function of temperature. The simulated results will be verified experimentally for the fabricated temperature sensor.

\section{ACKNOWLEDGEMENT}

My sincere gratitude and thanks goes to SRM Institute of Science and technology and Department of electronics and instrumentation for providing the sources and guidance. The authors would like to warmly thank Dr. P. Malar from Research institute \& Indian Institute of Technology, Bombay for helping the device fabrication and I would like to thank Dr. A Vimala Juliet and Mr. J Sam Jeba kumar for guidance and valuable suggestions and Special thanks go to MEMS design centre. Also, I would like to thank my friend Abdurrashid shuaibu Hassan for sharing his knowledge.

\section{REFERENCES}

1. Rong-Hua Ma; Yu-Hsiang Wang; Chia-Yen Lee; "Wireless Remote Weather Monitoring
System Based on MEMS Technologies", Sensors., 2011, 11, 2715-2727. 
2. Takayuki Fujita; Kazusuke Manenaka; "Integrated multi-environmental sensing system for the intelligent data carrier", Sensors and Actuators A: Physical., 2002, 97-98, 527-534.

3. Lee, C.Y.; "MEMS-Based Temperature control Systems for PCR Applications", Int. J. Nonlin. Sci. Num. Simulation., 2002, 3, 215-218.

4. SuyanXiao; Lufeng Che; Xinxin Li; Yuelin Wang; "A cost effective flexible MEMS technique for temperature sensing", Micro Electronics Journal., 2007, 38, 360-364.

5. Zelijko Hocenski; Ljubivoj Cvitas; Zelijko Lasinger; "Comparision of methods for nonlinearity correction of platinum resistance thermometer", SICE Annual Conference., 2008, 3151-3154.

6. Clifton L. Roozeboom; Bridget E. Hill; Vu Ang Hong; Chae Hyuck Ahn; Eldwin J.Ng; Yushi Yang; Thomas W.Kenny; "Multifunctional Integrated Sensors for Multiparameter Monitoring Applications", Journal of Micro Electro Mechanical Systems., 2015, 24, 810-821.

7. Edval J.P. Santos; Isabela B. Vasconcelos; "RTD based Smart Temperature Sensor: Process Development and Circuit Design", Microelectronics., 2008, 11-14.

8. Phatthanakun, R.; Deekla, P.; Pummara, W.; Sriphung, C.; Pantong, C.; Chomnawang, N.; "Design and Fabrication of Thin Film Aluminium
Microheater and Nickel Temperature Sensor", Nano Engineered and Molecular Systems., 2012, 112-115.

9. Bakker, A.; "CMOS Smart Temperature Sensor An Overview", Sensors., 2002, 1423-1427.

10. Zhen Fang; Zhan Zhao; Lidong Du; Jiangang Zhang; Cheng Pang; Daoqu Geng; "A portable Micro Weather Station", International Conference on Nano/Micro Engineered and Molecular systems., 2010, 379-382.

11. Huang, J.B.;Tung, S.; Ho C.M. Liu, C.;Tai, Y.C.; "Improved micro thermal shear-stress sensor", IEEE Transactions on Instrumentation and Measurement., 2006, 45, 570.

12. Rong-Hua Ma.; Dung-An Wang; Tzu-Han Hsueh; Chia-Yen Lee; " MEMS based flow rate and flow direction sensing platform with integrated temperature compensation scheme", Sensors., 2009, 9, 5460-5476.

13. Malar, P.; Zhao, J.; Van Kan, J.A.;"Fabrication of metallic stamps for injection moulding applications by combining proton beam writing and UV lithography", Applied Surface Science., 2012, 258, 4191-4194.

14. Sivasangari Sathiamoorthi.; Kunal, J.; Tiwari.; "Photo resist template fabrication and template assisted growth for surface patterning of technologically important $\mathrm{Cu}$ _2 ZnSnSe_2 thin films" Materials and Design., 2017, 127, 126-133. 